\title{
ExPLoIT: Exploiting Past Location Information and Transitivity for positioning in mobile sensor networks
}

\author{
Christophe Baraër, Kaustubh S. Phanse, Johan Nykvist and Luka Birsa \\ Department of Computer Science and Electrical Engineering \\ Luleå University of Technology \\ SE-971 87 Luleå, Sweden \\ \{christophe.baraer, kphanse\}@ltu.se; johan@csee.ltu.se; luka.birsa@gmail.com
}

\begin{abstract}
We present a novel distributed range-free technique called ExPLoIT for estimating geographical location of sensor nodes in mobile sensor networks. ExPLoIT is the first positioning technique that exploits location information at regular nodes in addition to using seeds. The key idea is the notion of confidence that a node has in its location estimate which can be used by other nodes for localization. Using Monte Carlo integration, a node estimates its current location and confidence based on its past location and the location information it receives from its neighbors. Node mobility is exploited to transitively localize the network. Our extensive simulation study shows that our proposal outperforms three other state-of-the-art range-free localization techniques under a wide range of conditions. The strength of ExPLoIT is that it is robust to irregular radio propagation, arbitrary seed and node density, and stochastic seed and node mobility; no special hardware is necessary at sensor nodes.
\end{abstract}

\section{INTRODUCTION}

Network localization or positioning - the ability to locate geographical position of network devices - is vital for wireless sensor networks. In many applications envisioned for these networks (e.g., target tracking, search and rescue, intruder detection), knowing the location of sensors is a prerequisite for the sensed data to be meaningful. Knowledge of sensor location can also be used to implement location-aware services such as geographical routing, energy efficiency algorithms, sensor coverage, and data aggregation.

Previous approaches to solving the localization problem generally rely on one or more of the following assumptions: a static network or nodes with controlled mobility; nodes equipped with special hardware for ranging; perfectly isotropic radio propagation; continuous connectivity to sufficient number of seeds (nodes that know their exact location, e.g., using GPS); large-enough network density; and planned deployment of sensor nodes.

In this paper, we revisit the localization problem with the goal of localizing sensors in a mobile sensor network where we assume a small number of seeds, but we make no assumption about the distribution of these seeds. The deployment of sensor nodes is arbitrary. The seed and node mobility is uncontrolled and the network may be intermittently connected, i.e., prone to network partitioning. Nodes are not equipped with special hardware for estimating their distance from other nodes or for estimating acceleration and direction of movement, and the radio propagation is anisotropic.

We propose ExPLoIT: a distributed range-free localization technique for mobile sensor networks that gets around these challenges by leveraging mobility and transitively spreading location information in the network. At a high level, the technique works as follows. ExPLoIT blurs the distinction between seeds and regular sensor nodes by empowering sensor nodes that are aware of their location to help other nodes localize. To implement this, we introduce the notion of confidence that a node has in its location estimate. As nodes move, they periodically exchange the 2-tuple of their location and confidence with neighboring nodes. Using this one-hop information and their own past location information, nodes estimate their current location and confidence. Nodes use Monte Carlo integration for this purpose. Initially, only seeds know their exact location (i.e., have a confidence of $100 \%)$. Regular sensor nodes are able to estimate their location as they move and hear seeds. Other nodes are able to estimate their location on hearing such location-aware nodes. This means that once seeds spread location information to parts of the network, other nodes can localize transitively without ever meeting a seed directly. Location-aware nodes age their confidence if no new knowledge is available, i.e., if they do not hear from other location-aware nodes.

Our proposed localization technique has the following salient characteristics.

1. To our knowledge, ExPLoIT is the first localization technique that enables regular nodes to track the accuracy of their location estimate and use it to help other nodes in the network. This is the power of ExPLoIT which makes it robust to low seed density, arbitrary spatial distribution of seeds, and uncontrolled seed and node mobility. It also greatly improves localization accuracy and stability, and enables faster convergence as compared to well-known state-of-the-art techniques.

2. ExPLoIT is fully distributed, local and "memoryful." It relies solely on one-hop information exchange with neighbors and nodes' own past location information. Network localization is then an emergent behavior as nodes move and spread 
location information transitively.

3. Only nodes with high enough localization accuracy help other nodes localize. In other words, nodes with ambiguous locations do not play any role in further localization. Exchange of location information being local and only nodes with high enough confidence contributing to network localization prevents localization error from spreading in the network.

4. The communication overhead and energy cost is bounded by the frequency of location updates. Further, gradually aging location information makes ExPLoIT tolerant to infrequent location updates.

5. EXPLOIT is robust to irregularities in radio propagation that may occur in reality making it more practical than other techniques that assume isotropic radio transmission.

The rest of the paper is organized as follows. In Section II, we review related work and give an overview of Monte Carlo integration (MCI). Next, we present our localization technique in Section III. Section IV describes our simulations and results comparing ExPLoIT with three other localization techniques. Finally, we present our concluding remarks in Section V.

\section{RELATED WORK AND BACKGROUND}

To better understand the characteristics and novelty of ExPLoIT, we begin by describing relevant techniques that have been previously proposed and explain how these techniques are different from our work. We then give an overview of Monte Carlo integration (MCI) as used in ExPLoIT.

\section{A. State-of-the-art}

Localization has been extensively studied for different network environments: from cellular networks, wireless LANs to wireless sensor networks and mobile ad-hoc networks. General surveys on localization can be found in [1]-[3]. Here, we review research most relevant to our work: localization in wireless sensor and ad-hoc networks.

Localization techniques can be broadly classified into two types: range-based and range-free. Range-based techniques measure signals such as angle of arrival (AoA) [4], received radio-signal strength [5] and time: time-of-arrival (ToA) [6] or time-difference-of-arrival (TDoA) [7], for estimating physical distance between network devices. Certain schemes [8] use a combination of signals, e.g., radio waves and ultrasound, for ranging. The drawback of range-based techniques is that the signal measurements are prone to errors due to perturbations from multipath fading, irregular signal propagation and node mobility, resulting in unreliable localization. To obtain a higher resolution, they often require special hardware (e.g., ultrasound device for TDoA, antenna arrays for AoA) that is expensive or impractical for extremely small sensor nodes, or they depend on unrealistic assumption (e.g., global time synchronization for ToA, isotropic radio propagation and idealistic path loss models for estimating distance between node pairs). We are interested in localization for mobile sensor networks using available radio capability and no special hardware at sensor nodes; hence, we adopt the range-free approach.
The range-free approach is based on using certain network characteristics for localization. In Centroid [9], each node estimates its location as the centre of mass of the location of seeds that it hears. If the placement of seeds is well planned, then Centroid can provide good location estimates. However, this is unrealistic in large-scale deployment of sensor networks and when movement of nodes and seeds is stochastic. APIT [10] divides the network into triangular regions between seeds and uses a grid algorithm to estimate the maximum likelihood of the region where a sensor node may be located. To attain high resolution in location estimate, APIT requires high density and uniform distribution of seeds.

DV-Hop [11] uses a strategy similar to distance-vector routing. Instead of one-hop broadcasts, seeds flood their location in the network periodically. Each node maintains a count of the number of hops to each seed from which it receives a message. Nodes estimate their location based on the location of seeds and the corresponding number of hops. Amorphous [12] adopts the same principle - that of using hop-count and seed locations to estimate node locations, but it uses off-line location estimation. Both DV-Hop and Amorphous assume isotropic radio propagation and require high node density for attaining high localization accuracy.

Tilak et al., [13] consider dynamic localization protocols for mobile sensor networks, but their focus is on predicting movement (direction and velocity) of sensor nodes to reduce the localization cost (energy). This work is complementary and can be used to enhance our proposed localization technique in the future.

The work in [14] also targets localization in mobile sensor networks. It adapts Monte Carlo Localization (MCL), commonly used for tracking mobile robots, to obtain the probabilistic distribution of a node's possible locations. Sequential Monte Carlo with importance sampling is used to estimate the posterior distribution of a node's location when it moves. Our work is closest in spirit to [14] (henceforth referred to as MCL), but there are significant differences. In [14], nodes solely depend on seeds for localization and thus require highenough seed density and high mobility (so that nodes meet seeds frequently); if a node does not meet any seed, then the node is unable to localize, causing ambiguity about its location and decreasing the overall accuracy of network localization. Further, nodes are not aware of the accuracy of their location estimate.

In proposing ExPLoIT we make several departures from previous research. Most importantly, no previous algorithm for absolute positioning considers using regular nodes (in addition to seeds) for localization. We present a novel notion of a node's confidence in its location that enables it to aid neighboring nodes in localization. This facilitates faster convergence and improved accuracy in network-wide localization under a wide range of network conditions. Our proposed technique is fully distributed, localized and exploits mobility for transitively spreading location information in the network. This makes it scalable for large-scale sensor networks. Using simulation, we show that ExPLoIT outperforms Centroid, Amorphous 
and MCL in terms of localization accuracy and stability under a wide range of conditions: stochastic seed and node mobility, different node and seed densities, and anisotropic radio coverage.

\section{B. Monte Carlo integration (MCI)}

In this section, we describe the basic idea of MCI as we use it in ExPLoIT. Monte Carlo methods are stochastic techniques, i.e., they are based on the use of random numbers and probability statistics to solve problems that are otherwise too complex to solve analytically.

In ExPLoIT, when a node hears one or more nodes, the overlapping area of the radio coverage of those nodes and the area bounding the node's potential location is used as an estimate of the node's confidence in its location estimate. We use perfectly circular radio coverage for this purpose; however, this does not compromise the effectiveness of ExPLoIT with realistic anisotropic radio coverage. Analytically calculating the overlapping area of two circles is fairly simple but solving the same problem with more than two circles is difficult and inefficient. Using MCI, we can estimate the overlapping area of an arbitrary number of circles. To understand the idea, let us explain the concept with only one circle. Consider a circle inscribed in a square-shaped dartboard. We throw a large number of darts $(N)$ onto the board and count the number of darts landing in the circle (success). If $A_{\text {bounding }}$ is the dartboard's area, then the circle's area $(A)$ is given by:

$$
A \approx\left(\frac{\text { success }}{N}\right) * A_{\text {bounding }}
$$

The area of the circle is approximately equal to the area of the square multiplied by the fraction of darts that landed in the circle. This is the fundamental concept of MCI. When dealing with two or more overlapping circles, success represents the number of darts landing in the overlapping area. We analyze MCI in more detail in Section III-B.

\section{LOCALIZATION TECHNIQUE}

In this section, we describe our localization technique for mobile sensor networks: Because nodes may move, we need to re-estimate their position periodically. For this purpose, we assume that time is divided into discrete time units, and localization is invoked once every time unit.

\section{A. Location estimation algorithm}

In ExPLoIT, the localization algorithm is designed to take advantage of location information of seeds as well as regular nodes. We introduce the novel idea of confidence that a node has in its location estimate. This is the first absolute positioning technique that enables regular nodes to keep track of their localization accuracy and use it to help other nodes localize. A node's confidence in its location estimate is expressed as:

$$
\text { Confidence }=1-\frac{A_{\text {over }}}{A_{\text {reference }}}
$$

For illustrative purposes, we choose a node's radio coverage area as the reference area $A_{\text {reference }}$ to define confidence.

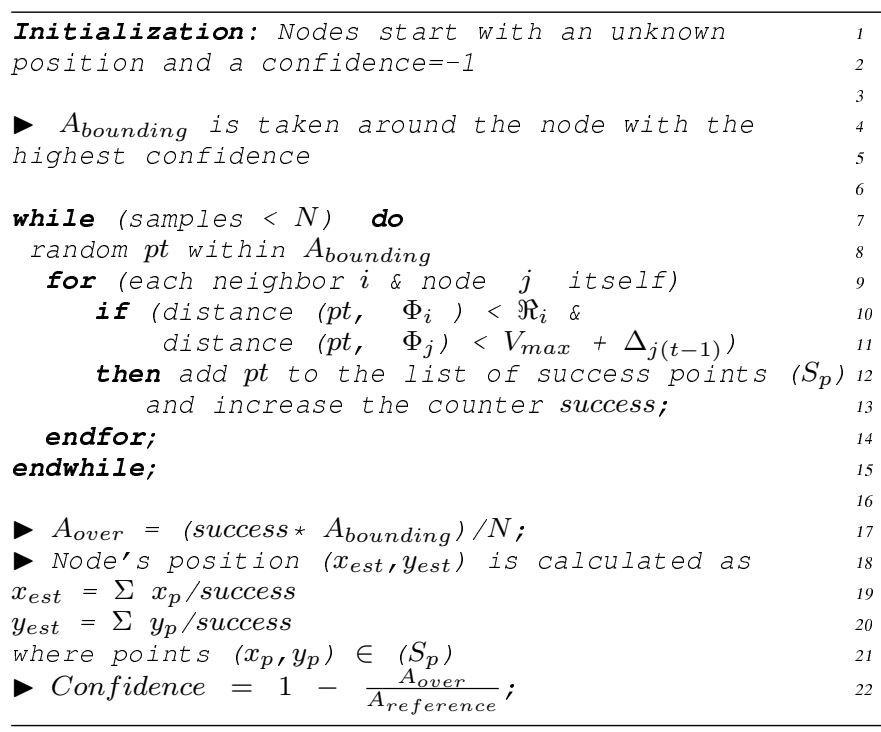

Fig. 1: ExPLoIT algorithm

$A_{\text {over }}$ is the overlapping area of the circular region bounding the node's possible current locations and the circular regions bounding the possible current positions of its neighbors and their radio coverage. A confidence of 1 (or 100\%) means that a node knows its exact location, e.g., seeds always have a confidence of $100 \%$. A confidence of zero suggests that the area bounding the node's potential current positions is equal to $A_{\text {reference }}$ (its radio coverage area). As a node moves, we calculate the region bounding its possible current positions at time unit $t$ as follows. We approximate $A_{\text {over }}$ from the previous time unit $(t-1)$ as area of a circular region with radius $\Delta_{(t-1)}$ which can be expressed as:

$$
\Delta=(1-\text { Confidence }) * r \quad \in[0 ; r]
$$

where $r$ is the radius of the node's circular radio coverage.

We assume that nodes are unaware of the direction and speed of their movement except that their speed is less than $V_{\max }$. Thus, when a node moves, the area bounding its possible current positions is a circle with radius $V_{\max }+$ $\Delta_{(t-1)}$ and the area bounding its current positions and radio coverage is a circle with radius $V_{\max }+\Delta_{(t-1)}+r$. For simplicity, we introduce the term pseudo-radius $\Re$.

$$
\Re=r+V_{\max }+\Delta
$$

Figure 1 gives an overview of the algorithm. Initially, a node has no knowledge about its position and its confidence is -1 . Nodes with a confidence of -1 do not help in the localization process. In other words, in the beginning, only seeds contribute to the localization process; nodes are able to localize only by meeting seeds. Then, as nodes might move, the location and confidence are updated at each time unit.

The algorithm works as follows. Let $\Phi_{i}$ denote the location estimate of node $i$ and $\Re_{i}$ denote the pseudo-radius of node $i$. Each iteration of the algorithm starts by determining the boundaries, $A_{\text {bounding }}$, in which MCI will be performed. This area is chosen around the node with the highest confidence 


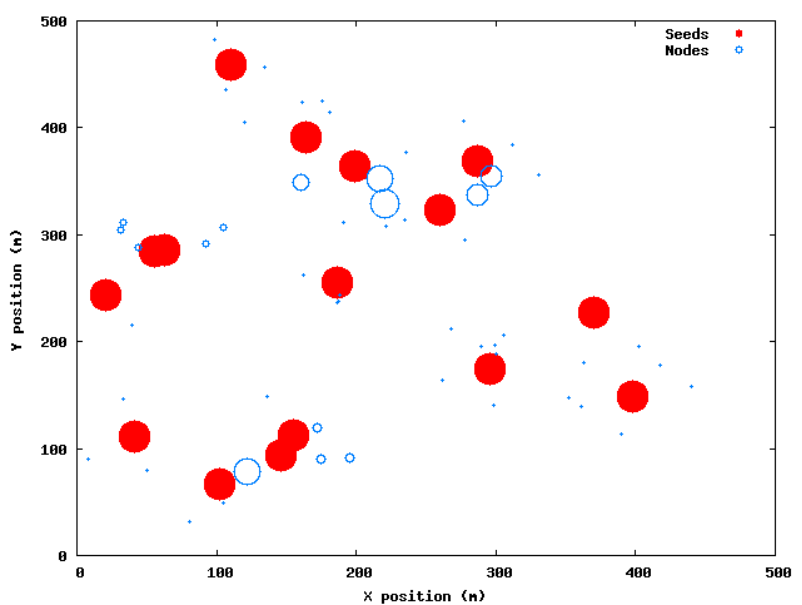

(a) Snapshot of the network at $t=1$

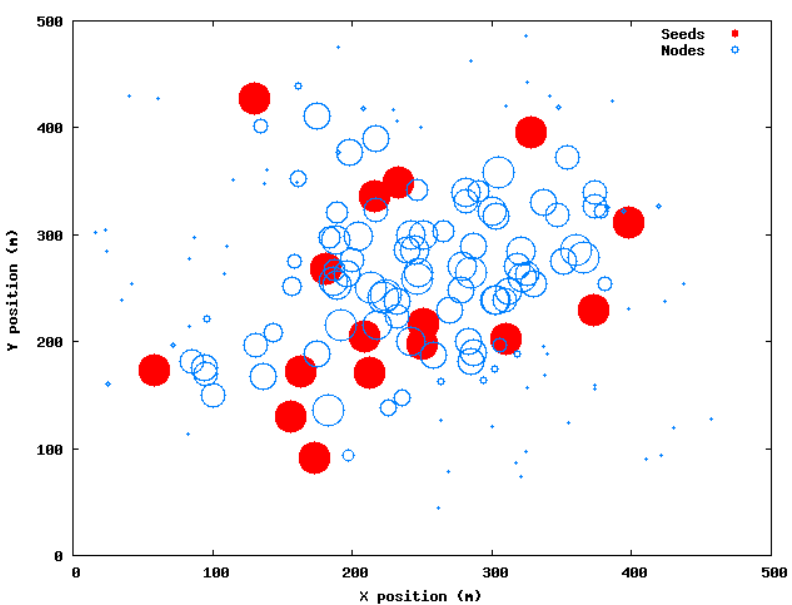

(b) Snapshot of the network at $t=31$

Fig. 2: Depiction of ExPLoIT.

with its last estimated location as center. If two or more nodes have the same highest confidence, the boundaries are randomly taken around one of those nodes. We elaborate on the reasons for choosing boundaries around the node with highest confidence in the next section. Given these boundaries, we now do random sampling (lines 7 to 15): we pick a random point within this area. If the point also happens to be in the overlapping area, then it is counted as a success. In MCI, the ratio of the number of success points to the total number of attempts or samples $(N)$ gives an estimation of the overlapping area $A_{\text {over }}$ (line 17). The location is estimated using the number of success points obtained during MCI sampling. We estimate the location of the node by computing the center of mass of all success points (lines 19 and 20).

During a certain time unit, if the node does not have neighbors, it keeps its latest estimated position and adds $V_{\max }$ to its pseudo-radius. The node calculates its new confidence using $\Delta_{t}=V_{\max }+\Delta_{(t-1)}$. However, once $\Delta_{t}$ exceeds $r$, i.e., the pseudo-radius $\Re$ exceeds twice the node's coverage radius $r$, the node's position is considered ambiguous and its confidence is demoted to -1 . While the node's confidence remains -1 , it does not contribute in the localization of other nodes.

Figure 2 illustrates ExPLoIT at work; only those nodes that have localized with a confidence equal to or greater than zero are shown. Shaded circles represent the seeds while unshaded circles represent regular nodes. The size of the circle represents the node's confidence in its location estimate: higher the confidence, bigger is the circle. In Figure 2a, we notice that initially (at time $t=1$ ), only few nodes close to the seeds are able to localize with a high confidence. Other nodes, shown as specks, are localized but have a low confidence close to zero. With time, nodes cooperate and help other nodes localize. As location information spreads in the network, more nodes have a high confidence. A snapshot of the network at time $t=31$ is shown in Figure 2b.

\section{B. Analysis}

MCI is statistical by nature. Based on the one-hop neighborhood information a node receives, it can estimate the overlapping area using MCI, given by Equation (1). Such a hit/miss experiment follows a binomial distribution with a probability of success, where success is the event of a point landing in the overlapping area, $\mathbb{P}\{$ success $\}$ is:

$$
\mathbb{P}\{\text { success }\}=\frac{A_{\text {over }}}{A_{\text {bounding }}}
$$

The expected value of the number of successes after $N$ thrown is:

$$
\mathbb{E}\left(N_{\text {success }}\right)=N * \mathbb{P}\{\text { success }\}=N * \frac{A_{\text {over }}}{A_{\text {bounding }}}
$$

and the variance is:

$$
\operatorname{Var}\left(N_{\text {success }}\right)=N * \mathbb{P}\{\text { success }\} *(1-\mathbb{P}\{\text { success }\})
$$

The estimator of the overlapping area is $\widehat{A}_{\text {over }}=N_{\text {success }} *$ $\frac{A_{\text {bounding }}}{N}$. Its expected value is:

$$
\begin{aligned}
\mathbb{E}\left(\widehat{A}_{\text {over }}\right) & =\mathbb{E}\left(N_{\text {success }}\right) * \frac{A_{\text {bounding }}}{N} \\
& =N * \frac{A_{\text {over }}}{A_{\text {bounding }}} * \frac{A_{\text {bounding }}}{N} \\
& =A_{\text {over }}
\end{aligned}
$$

$\widehat{A}_{\text {over }}$ is an unbiased estimator of $A_{\text {over }}$ whatever the size of $N$. The variance, $\operatorname{Var}\left(\widehat{A}_{\text {over }}\right)$, of the estimator is given by:

$$
\begin{aligned}
\operatorname{Var}\left(\widehat{A}_{\text {over }}\right) & =\operatorname{Var}\left(N_{\text {success }}\right) *\left(\frac{A_{\text {bounding }}}{N}\right)^{2} \\
& =\frac{A_{\text {over }}}{N} *\left(A_{\text {bounding }}-A_{\text {over }}\right)
\end{aligned}
$$

Due to the law of large numbers, [15], if $N$ is sufficently large, $\widehat{A}_{\text {over }}$ has a gaussian distribution with mean $\mu=A_{\text {over }}$ 
and $\sigma=\frac{A_{\text {over }}}{N} *\left(A_{\text {bounding }}-A_{\text {over }}\right)$ that it is common to write as:

$$
\widehat{A}_{\text {over }} \sim \operatorname{Norm}\left(A_{\text {over }}, \frac{A_{\text {over }}}{N} *\left(A_{\text {bounding }}-A_{\text {over }}\right)\right)
$$

From Equation (13), we note that for a fixed number of samples $N$, the variance gets smaller for smaller $A_{\text {bounding }}$. This explains our choice of choosing the boundaries for MCI sampling around the node with the highest confidence. As shown in Figure 3, for the same number of samples, the variance will be smaller for the MCI boundary chosen in the figure on the left than the one on the right. We can also reduce the variance by increasing the number of samples. The convergence rate, i.e., how quickly the error decreases with the number of samples in MCI is proportional to $\sqrt{\frac{1}{N}}$ where $N$ is the number of samples. This means that quadrupling the number of samples will halve the error.
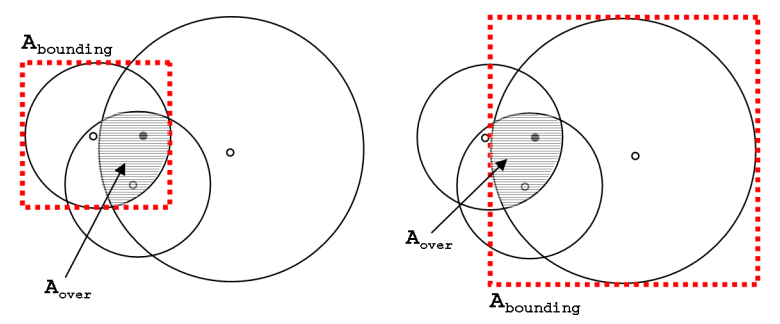

Fig. 3: Boundary boxes for MCI

\section{Evaluation}

In this section, we present our simulation results characterizing ExPLoIT and compare its performance with that of three other range-free localization techniques: Centroid, Amorphous and MCL.

\section{A. Simulation parameters}

The simulator from [14] was used for our simulations; we extended the simulator to implement ExPLoIT ${ }^{1}$. In all scenarios, we begin by uniformly distributing nodes in an area of 500 x 500 sq.m. Unless otherwise specified, we consider a network with 320 nodes including 32 seeds. We used a modified version of the random-waypoint (RWP) mobility model that fixes the known problems of the original RWP model, namely the decay of average speed [16] and gathering of nodes near the centre of the simulation area [17] during simulation. In the modified RWP model, a node randomly chooses a destination point, its speed uniformly selected from [0; $\left.V_{\max }\right]$, where $V_{\max }$ represents the maximum speed of mobile nodes. The speed of nodes can change before they reach the chosen destination, unlike the original RWP model. The pause time between each movement is set to 0 , so the average speed is exactly $V_{\max } / 2$. The nodes remain uniformly distributed during the simulation.

For all nodes, we set the transmission range $(r)$ to $50 \mathrm{~m}$, and set $V_{\max }$ to $0.2 r$. Initially, we use perfectly circular isotropic

\footnotetext{
${ }^{1}$ Available at: http://www.sm.luth.se/kphanse/ExPLoIT/
}

radio pattern, but later, in Section IV-E, we use more realistic irregular radio patterns. The simulation results are an average of five independent simulation runs conducted using different pseudorandom numbers as seed values. Each simulation runs for at least 100 time units.

The network parameters we vary are as follows:

- Network density: represents the number of nodes in the network for the given simulation area. We track the neighbor count that gives us an idea of how dense is the network. The neighbor count is defined as [18]:

$$
\text { Neighbor Count }=\frac{\pi * r^{2}}{\frac{h * w}{n}}
$$

where $h$ and $w$ is respectively the height and width of simulation region, $r$ is the transmission range and $n$ is the number of nodes in the network.

- Seed density: the percentage of seeds present in the network.

- Mobility and speed: of seeds $\left(V S_{\max }\right)$ and nodes $\left(V N_{\max }\right)$.

Our primary metric of interest is the location estimate error expressed in terms of the node coverage radius $r$. The vertical bars plotted in each figure represent the standard deviation.

\section{B. Impact of network density}

In Figure 4, we can observe the behavior of the different localization techniques in presence of different network densities. In general, two phases are noticed in the localization process: the initialization phase and the stable phase. During the initialization phase, the localization error decreases as more and more nodes localize. In the stable phase, the average localization error for the network reaches an equilibrium with some fluctuation. In all four cases, ExPLoIT outperforms the other techniques - it is the quickest to reach the stable phase and has the lowest average localization error. In ExPLoIT, by exchanging their location estimate and confidence, nodes quickly spread location information throughout the network. Further, in sparse networks (Figures $4 \mathrm{c}$ and $4 \mathrm{~d}$ ), it results in relatively stable localization even when the network is prone to partitioning,

In a dense network (Figure 4a), Centroid performs worse than Amorphous and MCL due to the low seed density of $10 \%$. Amorphous is almost as quick as ExPLoIT in reaching the stable phase for a highly dense network. However, unlike MCL and ExPLoIT, Amorphous and Centroid are memoryless techniques, in that they do not exploit past location information. Hence, they do not improve over time. The strength of Amorphous is its ability to aggregate location information from a node's multi-hop distance to at least three seeds. However, this makes it highly sensitive to network density. As the neighbor count decreases, Amorphous suffers from high average localization error and dramatic fluctuations in the localization error due to lack of reliable estimates of distance to seeds. In fact, once the neighbor count is below 3 (Figure 4c), both Centroid and Amorphous fail to stabilize as the localizationn error oscillates considerably after the 


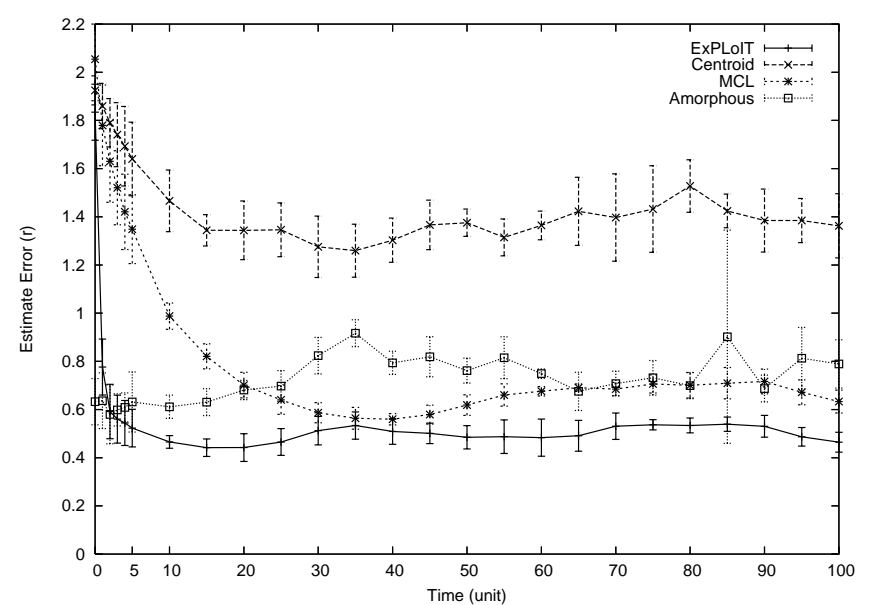

(a) Total of 320 nodes, $10 \%$ seed, Neighbor count $\simeq \mathbf{1 0}$

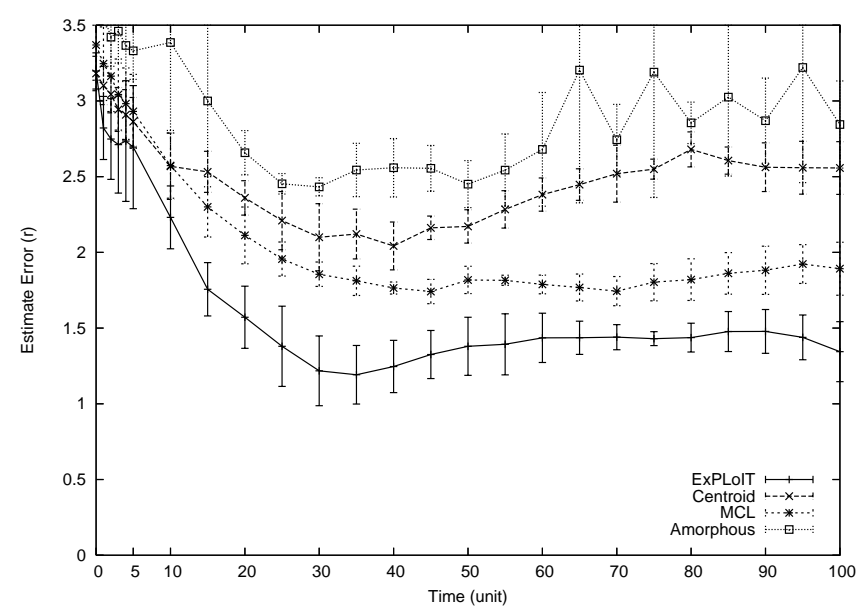

(c) Total of 80 nodes, $10 \%$ seed, Neighbor count $\simeq \mathbf{2 . 5}$

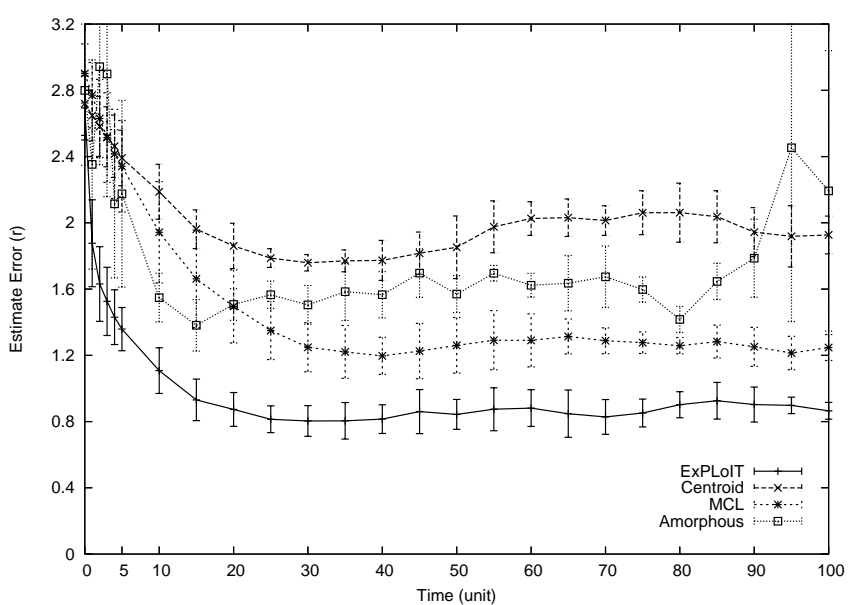

(b) Total of 160 nodes, $10 \%$ seed, Neighbor count $\simeq \mathbf{5}$

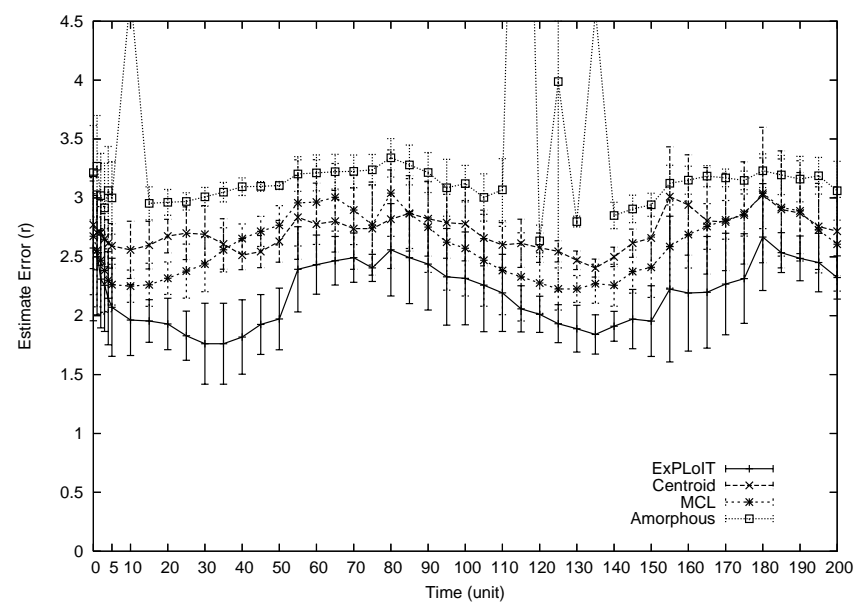

(d) Total of 40 nodes, $10 \%$ seed, Neighbor count $\simeq \mathbf{1 . 2}$

Fig. 4: Impact of network density. $r=50 m, V_{\max }=0.2 r$

initialization phase. When the network is extremely sparse (Figure 4d), nodes meet infrequently and even the past location information timesout. We purposely plot the results over 200 time units to observe the oscillations; the behavior seems to repeat every 100 time units. Exploiting location awareness at nodes, ExPLoIT still gives the best performance.

To conserve battery power, sensor nodes may choose to participate in localization less frequently. This has similar effect on the overall network localization accuracy as low network density. Hence, we do not show those results here.

\section{Seed density}

Here, we study how the various localization techniques perform under different seed densities. The results are shown in Figure 5. ExPLoIT performs best at extremely low seed density (less than 2\%). After that, given the high network density (neighbor count $\simeq 10$ ), Amorphous's multihop strategy gives it better performance. However, Amorphous is memoryless and suffers from cumulative localization error over multiple hops; this limits its accuracy and it does not see any improvement beyond 6\% seed density. ExPLoIT and MCL, on the other hand, continually improve; their performance converges beyond $10 \%$ seed density. Centroid performs worst for low seed density as nodes are unable to hear simultaneously from at least three seeds.

\section{Mobility}

Figure 6 shows the impact of the speed of nodes (including seeds) on the performance of the various localization techniques. The maximum speed $\left(V_{\max }\right)$ ranges from $0.1 \mathrm{r} \mathrm{m} / \mathrm{time}-$ unit to $2 r \mathrm{~m} /$ time-unit. ExPLoIT outperforms other techniques at reasonable speeds. The accuracy of Centroid, Amorphous and ExPLoIT is mostly unaffected by change in node speed; though, ExPLoIT 's performance degrades slightly at very high speeds because the confidence of nodes ages quickly at high speeds. MCL's accuracy is a function of how frequently nodes meet seeds; hence, MCL performs poorly at low mobility and its accuracy improves with increase in node speed.

We then consider a network where seeds are static and only regular nodes are mobile. The results are shown in Figure 


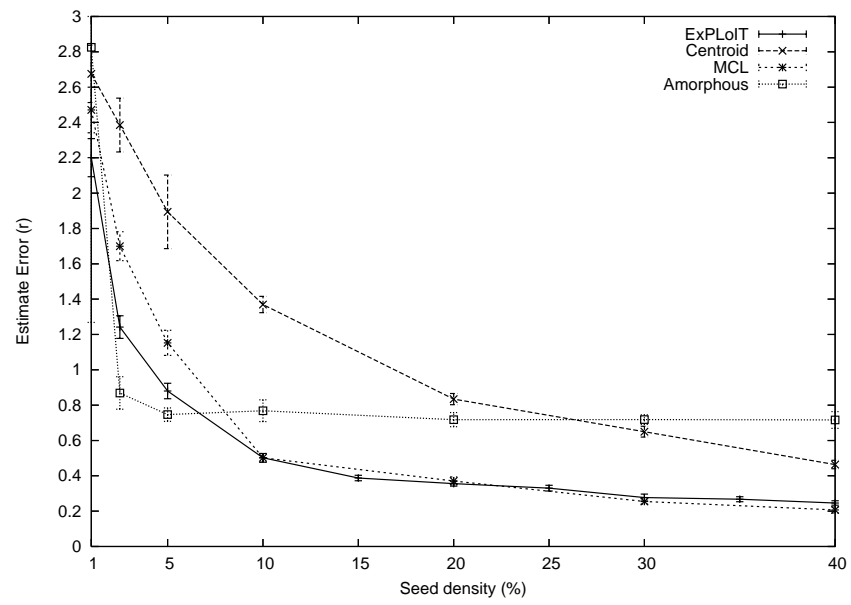

Fig. 5: Impact of seed density. Total of 320 nodes, $V_{\max }=$ $0.2 r, r=50 m$

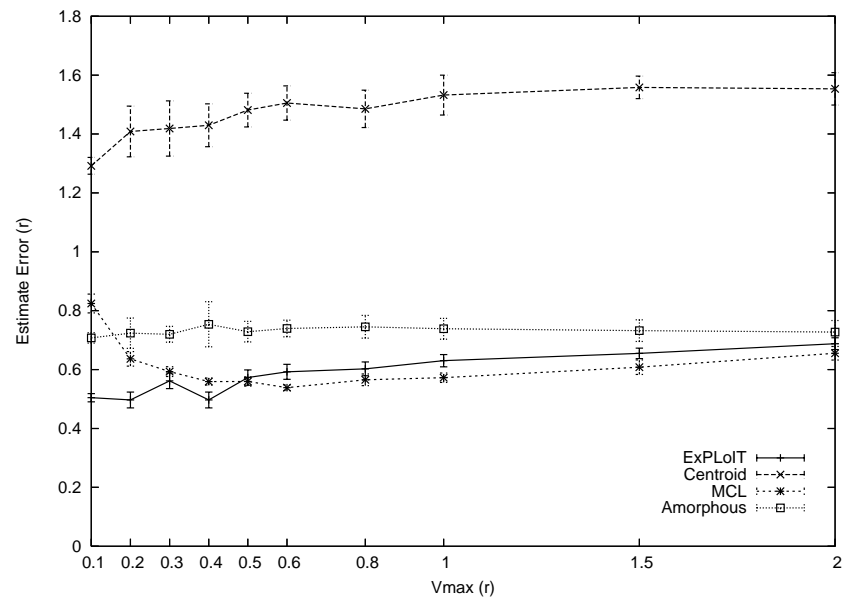

Fig. 6: Impact of speed. Total of 320 nodes, $10 \%$ seed, $r=$ $50 m$

7. We observe that, as compared to Figure $4 \mathrm{a}$ (where both nodes and seeds are mobile), MCL's localization accuracy degrades considerably. Not only does MCL take much longer to converge to the stable phase, but its average localization error is almost twice that in Figure 4a. On the other hand, ExPLoIT is unaffected by static seeds and performs better than other techniques both in terms of accuracy and reliability (stability and standard deviation).

\section{E. Irregular radio transmission}

So far we used nodes with isotropic circular transmission with radius $r$. In practice, however, radio transmissions are anisotropic and radio range may vary significantly with environmental conditions and antenna imperfections; these variations may impact the localization accuracy. To simulate this, we use degree of irregularity (DOI). For example, a $D O I=0.3$ denotes a radio range that varies randomly in each direction, the range chosen from $[0.7 r, 1.3 r]$.

Figure 8 shows the localization error as a function of DOI. Amorphous is the most sensitive to radio range variation and its localization error increases for $D O I>0.1$. The

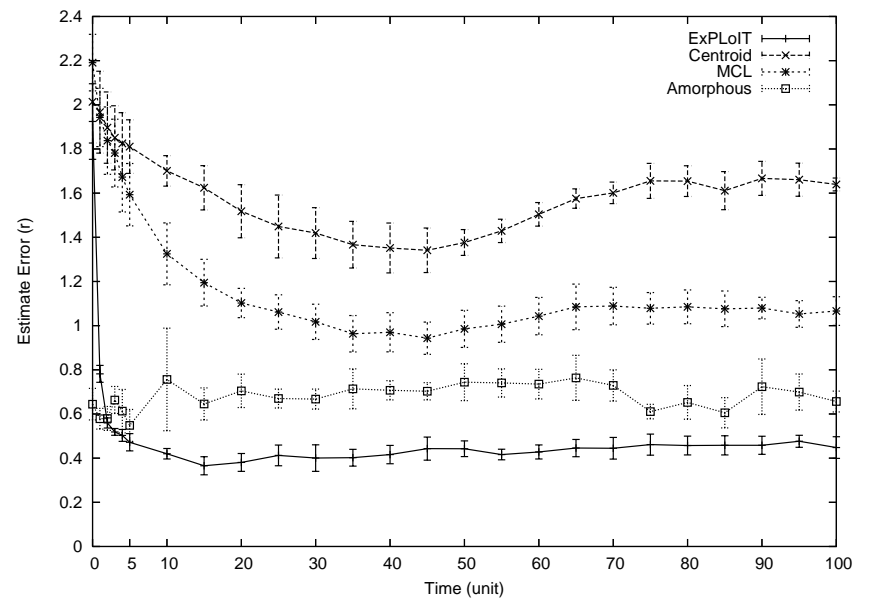

Fig. 7: Impact of mobility. Fixed seeds, mobile nodes, $r=$ $50 m, V N_{\max }=0.2 r, V S_{\max }=0$

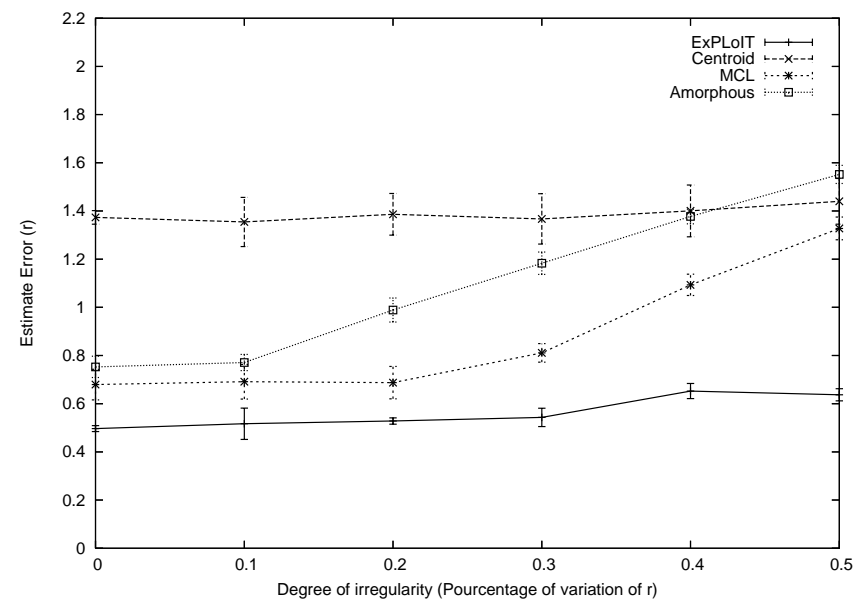

Fig. 8: Impact of irregularity. Total of 320 nodes, $10 \%$ seed, $r=50 m, V_{\max }=0.2 r$

hop counting in Amorphous assumes perfectly circular radio transmissions; this leads to inconsistent hop counts as the ranging error gets accumulated over several hops for higher DOI. MCL is robust to radio range variations up to DOI = 0.3 , but in extreme conditions of DOI (equal to and greater than 0.4), MCL's localization error increases. In MCL, nodes use seeds within two hops to localize. For high DOI, this inserts sampling errors over two hops. ExPLoIT and Centroid, on the other hand, are local techniques and nodes estimate the location as the centre from the locations of their onehop neighbors (only seeds in case of Centroid). Aggregating location information from one-hop neighbors helps ExPLoIT and Centroid abate the effects of DOI.

\section{F. Number of samples}

Increasing the number of samples in Monte Carlo integration can improve the localization accuracy in ExPLoIT at the cost of additional computing. In our simulations, we observed that beyond the threshold of 500 samples, increasing the number of samples did not improve ExPLoIT 's accuracy. This threshold is determined by the average ratio of the overlapping 


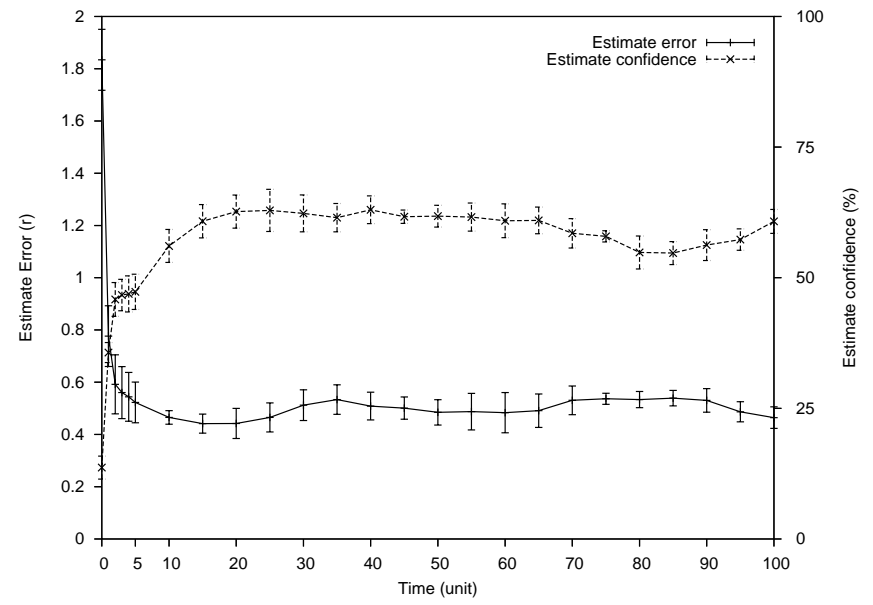

Fig. 9: Relation between the average confidence in the network and localization error

area $\left(A_{\text {over }}\right)$ to the bounding area $\left(A_{\text {bounding }}\right)$ : smaller the ratio, larger will be the threshold (number of samples).

\section{G. Confidence}

Figure 9 shows an interesting relation where the location estimate error is a mirror image of the confidence that nodes have in their estimate. The y-axis on the left side represents the average localization error, and the y-axis on the right side represents the average confidence. As the location information spreads in the network and the nodes become more confident, the overall localization accuracy improves. The initial confidence is non-zero as seeds always know their location and have a confidence of $100 \%$.

\section{Conclusion}

This paper revisited the network localization problem in the context of mobile sensor networks. The main contribution of this work is the idea of exploiting location information at regular nodes in addition to seeds; we introduced the notion of confidence that allows nodes to track their localization accuracy and use it to help neighboring nodes localize. We illustrated the benefits of doing this using our proposed rangefree localization technique ExPLoIT.

Our simulation study showed that ExPLoIT outperforms three other range-free localization techniques: Amorphous, Centroid and MCL, in terms of localization accuracy as well as stability under a wide range of conditions. ExPLoIT adapts well to varying network and seed density, static seeds, speed of node movement, and importantly, copes with randomly varying radio range. The robustness of ExPLoIT to anisotropic radio propagation makes it a prime candidate for practical use in real networks. ExPLoIT operates locally as nodes exchange location information only with their neighbors; hence, it scales well-the overhead bounded by the frequency of location updates - and prevents localization error from spreading.

Simulations also showed a direct relation between the confidence nodes have in their location estimate and the overall network localization accuracy. Given the benefits of the notion of confidence, we hope that this basic idea of ours will serve as a guide for future developments in the area of network localization. In its current form, ExPLoIT approximates the region bounding a node's potential positions as a circle. In the future, the performance can be optimized by modeling this region as a general polygonal shape.

\section{ACKNOWLEDGMENT}

The authors would like to thank Håkan Jonsson for valuable discussions about this work. This research was partly supported by the Vinnväxt ProcessIT Innovation programme and the EU Mål-1 project C4-DTN.

\section{REFERENCES}

[1] J. Hightower and G. Borriello. Location systems for ubiquitous computing. IEEE Computer, 34(8), August 2001.

[2] G. Sun, J. Chen, W. Guo, and K. Liu. Signal processing techniques in network-aided positioning: a survey of state-of-the-art positioning designs. IEEE Signal Processing Magazine, 22(4):12-23, aa 2005.

[3] N. Patwari, J. Ash, S. Kyperountas, R. Moses, and N. Correal. Locating the nodes: Cooperative localization in wireless sensor networks. IEEE Signal Processing Magazine, 22(4):54-69, July 2005.

[4] D. Niculescu and B. Nath. Ad hoc positioning system (aps) using aoa. In IEEE INFOCOM, April 2003.

[5] P. Bahl and V. Padmanabhan. Radar: An in-building rf-based user location and tracking system. In IEEE INFOCOM, volume 2, pages 775-784, March 2000.

[6] R. L. Moses, D. Krishnamurthy, and R. Patterson. A self-localization method for wireless sensor networks. EURASIP Journal on Applied Signal Processing, 4:348-358, March 2003.

[7] N. Priyantha, A. Chakraborty, and H. Balakrishnan. The cricket locationsupport system. In ACM MOBICOM, pages 32-43, 2000.

[8] N. Priyantha, H. Balakrishnan, E. Demaine, and S. Teller. Mobile assisted localization in wireless sensor networks. In IEEE INFOCOM, volume 1, pages 172-183, March 2005.

[9] N. Bulusu, J. Heidemann, and D. Estrin. Gps-less low cost outdoor localization for very small devices. IEEE Personal Communications Magazine, 7(5):28-34, October 2000.

[10] T. He, C. Huang, B. M. Blum, J. A. Stankovic, and T. Abdelzaher. Range-free localization schemes for large scale sensor networks. In ACM MOBICOM, pages 81-95, 2003.

[11] D. Niculescu and B. Nath. Dv based positioning in ad hoc networks. Kluver Journal of Telecommunication Systems, 22(1-4):267-280, July 2003.

[12] R. Nagpal, H. Shrobe, and J. Bachrach. Organizing a global coordinate system from local information on an ad-hoc sensor network. In International Workshop on Information Processing in Sensor Networks, April 2003.

[13] S. Tilak, V. Kolar, N. B. Abu-Ghazaleh, and K-D. Kang. Dynamic localization protocols for mobile sensor networks. In IEEE International Workshop on Energy Efficiency in Ad Hoc and Sensor Networks, pages 587-592, April 2005.

[14] L. Hu and D. Evans. Localization for mobile sensor networks. In ACM Mobicom, pages 45-57, September 2004.

[15] W. Feller. An Introduction to Probability Theory and Its Applications, chapter Laws of Large Numbers, pages 228-247. Wiley, New York, third edition, 1968.

[16] J. Yoon, M. Liu, and B. Noble. Random waypoint considered harmful. In IEEE INFOCOM, volume 2, pages 1312-1321, 2003.

[17] C. Bettstetter, G. Resta, and P. Santi. The node distribution of the random waypoint mobility model for wireless ad hoc networks. IEEE Transactions on Mobile Computing, 2(3):257-269, July-Sept 2003.

[18] S. Kurkowski, T. Camp, and M. Colagrosso. Manet simulation studies: the incredibles. ACM SIGMOBILE Mobile Computing and Communication Review, 9(4):50-61, October 2005. 